\title{
A review on digital mental health applications types, PsycappsE and eQuoo
}

\author{
Silja Litvin
}

Health Foundry, 1 Royal Street, SE1 7LL London, UK

\begin{abstract}
With the World Health Organisation (WHO) stating that one in four are struggling with mental health issues, self-harm rising by $68 \%$ in young adults since 2011 and only $35 \%$ of the affected population receiving treatment, carers, insurers and mental health professionals have been looking for scalable solutions, specifically for digital interventions. As the start-up industry develops more supporting ecosystems for researchers to develop innovative digital solutions, thousands of mental health apps have been published since 2016 all vying for customers and partnerships. This is a short review on mental health app categories and app development with PsycAppsE and eQuoo in focus.
\end{abstract}

\section{Introduction}

An overview of the 'Top 50' in the Google and iOS Apps Stores listed existing mental health applications (from now on mHealth apps) shows that most published apps can be traced back to two different developer sources: Individuals that have experienced a mental health issue in the past and decided to develop a resource for others and researchers developing tools originating out of their findings. While the former struggle with developing tools that are evidence-based, the latter find themselves struggling with user retention and user acceptancy [1]. The mHealth apps that are the most successful as defined by traction and funding are those where founders have integrated researchers early on and where researchers partnered with marketers and UX designers [2].

A simple real-time selection was conducted on June 12th, 2019 by assessing the top $50 \mathrm{mHealth}$ apps in the Google Play store using the key words 'mental health apps'. They were then categorised into 4 different groups according to the services they offer. Yielding to the restrictions of a short review, only the two highest ranking mHealth apps were reviewed for each group:

\section{Categorisation of Mhealth apps}

\section{Marketplace}

Location, time and financial restrictions are the most commonly named thresholds to finding and adhering to face-to-face therapy [3]. Betterhelp and Talkspace are the best funded video and chat therapy apps, with Talkspace closing a \$50 million financing round in June 2019 [4-6]. While video therapy is effective, certain risks come along with it such as the reduction of the therapeutic alliance $[7,8]$. Marketplace solutions aren't endlessly scalable, as accredited psychologists need to be willing to call and chat for very low renumeration and for sessions as short as 15 minutes, which leads many therapists to question its ethics [9]. Betterhelp has recently been involved in a scandal as users became aware that not all of the therapists they were chatting to were accredited [10].

Authors conclusion - Pros: Marketplace mHealth apps - low barrier to entry. Cons: Decline of therapeutic alliance, potential exposure to non-accredited or properly trained individuals offering service and insufficient session duration.

\section{Mindfulness apps}

Calm and Headspace have been extremely successful mindfulness apps, with Calm being named the first mindfulness 'Unicorn' and over $\$ 88$ million funding [11-13]. While Calm is extremely popular, Headspace has onboarded a research team lead by Dr. Megan Jones Bell and is conducting clinical trials around Healthcare resource use (e.g., healthcare utilisation and costs), anxiety, Type 2 diabetes, cardiovascular disease, chronic pain, autoimmune disorders, sleep and sleep quality, medical error, business revenue (e.g., sales revenue) and performance, sports performance and mindfulness in education/ schools [14]. To date, 16 clinical trials from non-affiliated research teams have been conducted showing significant effects in wellbeing, stress management, compassion and irritability [15]. While mindfulness is not a lone-standing intervention, it is often used within therapy as a self-management and regulation tool [16].

Authors conclusion - Pros: Mindfulness apps - significant effect on multiple dimensions of psychological well-being, affordable. Cons: Not interchangeable with therapeutic interventions.

\section{Chatbot apps}

With the advancement of Artificial Intelligence (AI), natural language processing (NLP) has led to an explosion of chatbots in every field of human communication [17]. As face-too-face therapy is comprehensively composed of conversation, developers have been

${ }^{*}$ Correspondence to: Silja Litvin, Health Foundry, 1 Royal Street, SE1 7LL London, UK, E-mail: silja@psycapps.com

Key words: Mental health, mental health apps (mHealth apps), digital therapy, mobile therapy, evidence-based apps

Received: June 22, 2019; Accepted: July 02, 2019; Published: July 05, 2019 
looking into how chatbots may facilitate therapeutic interventions via chat. Stanford originating chatbot, Woebot showed significant decrease in depression and anxiety symptoms in a short trial over two weeks $[18,19]$. Chatbot Tess developed by non-clinician Michiel Rauws showed similar results in a four-week trial $[20,21]$. Both chatbots are founded on CBT lessons with psycho-educational content.

Authors Conclusion - Pros: Chatbot Therapy Apps - low barrier to entry and evidence-based mobile intervention. Cons: Despite being a chatbot, both Woebot and Tess are heavily scripted, making it less of a real AI and more of a highly sophisticated decision tree. NLP requires the bot to pick up and integrate language from the human conversation side. With a clinical population that could have devastating effects as Microsoft's chatbot experiment clearly showed, making a safe clinical AI bot a thing of the future[22].

\section{Mobile therapy apps}

Mobile therapy apps build on the success of evidence-based web interventions, moving to a mobile platform, following the trend from web to mobile $[23,24]$. Most mHealth apps of this type transfer the content of face-to-face therapy into digital, such as CBT, DBT and psychoeducation [25]. The shortcomings of this strategy is that the biggest influence on therapy outcome, the therapeutic alliance is excluded by design [26]. This together with stigma, lack of evidence, unstructured app store rankings and the lack of motivation for self-help has led to a cornucopia of mental health apps trying to offer scalable interventions, none of which have established market dominance [27,28]. Moodpath and Pacifica appear to be leading with the most users as can be assessed through existing sources [29,30]. Pacifica is empirically based and will be publishing the results of an ongoing trial within the next few months [31]. Moodpath is similarly empirically based and has four on-going clinical trials with non-affiliated research teams, no published content yet. PsycAppsE, an evidence-based mHealth App that went through an extensive clinical trial showed a significant decrease of depression and a decrease in anxiety but struggled with attrition as most mHealth apps do $[32,33]$.

Authors Conclusion -Pros: Mobile therapy apps - affordable, low barrier to entry, effective treatment if evidence based. Cons: High attrition rates, lack of motivation of clients, few evidence-based products, confusion within clients on how to choose the right mHealth App.

\section{Market Needs and Limitations}

Research shows that most mHealth apps struggle with attrition, having most clients jump off before an app can show effect [34]. This has various reasons, the missing therapeutic alliance and lack of engaging content and design being the leading hypothesis. This leads to the market still lacking mHealth apps that combine:
1) Effectiveness
2) Evidence-based research
3) 'Stickiness'

\section{The making of an App: The development of PsycAppsE The design}

The development of PsycAppsE begun early 2016 with extensive research of existing mHealth products and web-based interventions. In 2016, there were less than 50 mental health apps on the iTunes App Store, only one which had conducted a clinical trial (Superbetter). Together with a graphic designer and app developer the user interface was designed, content was chosen based on CBT, and positive psychology and psychoeducation was found in the research of successful web-based interventions. The wireframes were mapped out using customary paths, buttons and icons to ensure a smooth and intuitive user experience. Methods such as psychoeducation, self-assessment, journaling, selfmanagement, and goal-setting were designed to fit a mobile app.

\section{The Development}

A US based software engineer then developed the backend and connected all interfaces with commands, each development step being iteratively tested for functionality and program errors called bugs. The language used was $\mathrm{C}+$ and the backend was developed on an FTP server. Due to the Apple programming language being new to the developer, it took 3000 hours building and testing the app. Financial restrictions of the researching party led to PsycAppsE being developed only on iOS instead of multi-platform.

\section{The Research}

Recruited through platforms, such as mTurk, Prolific, the LMU University Newsletter and Social Media, 276 participants partook in a randomised control trial (137 in the test group, 139 in the control group), both groups were tested using the Beck Depression Inventory (BDI), Life Satisfaction Scale, and a one-item Likert Scale for anxiety at the pre- and post-evaluation phases within a four week period. The test group used the app, while the control group filled out a digital survey with no further intervention.

\section{App Findings}

Results of repeated measures of ANOVAs showed statistically significant increases when using the app over a timeframe of four weeks. The app significantly lowered the test group's depression levels, measured by the BDI as .57. While not significant, anxiety levels were lowered by .19 .

\section{Conclusion}

While the app showed a significant effect on depression and a lowering of anxiety scores, the attrition rate was still alarming, with only $55 \%$ of the participants completing the 4 -week trial. In comparison with conventional mHealth attrition rates of 70-90\% [35]. PsycAppsE still stands out positively, but there was a $€ 5$ incentive, indicating that attrition rates with commercial clients would be higher. With regards to promising outcomes of apps using gamification as a motivator, and the overall positive effect of play on well-being, it was concluded that gamifying mHealth interventions could be the way to ensure 'stickyness', acting as a carrier of the intervention methods $[36,37]$.

\section{The Development of Equoo, the Emotional Fitness Game}

\section{Learnings from PsycAppsE}

The chief lesson the researchers of PsycAppsE took away after analysing their results and looking at meta studies concerning the effectiveness of mobile intervention apps was that digital transformation of evidence-based face-to-face interventions is not sufficient to be an effective intervention without onboarding a compelling motivational factor. A vulnerable population, and even more so, a clinical one, additionally struggles with lack of drive, curiosity, motivation and cognitive impairment making them less likely to self-encourage adherence to mobile interventions [38]. 


\section{Gaming as Carrier}

In pharmaceutics, successful drug administration is dependent on finding and implementing the right drug carrier to improve the delivery and the effectiveness of drugs [39]. This applies for antidepressants and other mood disorder treatments and can be considered just as important as the effective ingredients. One could use drug carriers as an analogy for the form in which therapy is administered, the Therapeutic Alliance being a time-tested success. With stand-alone mobile therapies, studies indicate that gamifying therapy could be a potentially robust carrier to support the delivery and effectiveness of the intervention [35]. Playing games is well known to have a positive effect on well-being and serious games are slowly starting to raise awareness in the world of psychology and psychiatry, making gaming a logical choice [40].

\section{The development of eQuoo}

After partnering with a game developing company that had a 15year track record in developing games from franchised movie successes, the research team behind PsycAppsE developed eQuoo, the Emotional Fitness Game targeting 18-28 year olds [41]. Utilizing psychoeducation, storytelling, elements of CBT and positive psychology, 10 psychological skills relevant to depression and anxiety were chosen to be administered in a 5-week course. In each course, two skills are taught using an avatar, Dr Joy and two characters, Jasmin and Noah in a playful tutorial. Once the skills have been successfully understood as queried in a 1 item multiple choice test, a choose-your-own-adventure story opens where the player needs to implement the learned skills in order to collect coins and level up. The safe, because fictional, yet stimulating environment allows the player to practice the skills, embedding them into their memory [40]. With an average session time of 13 minutes spent by the $35 \mathrm{k}$ plus players that have downloaded the free-to-play game, since the launch in July 2018, and $17 \%$ of their players finishing the 5 week course, eQuoo by far supersedes the on average $2-6 \%$ adherence for current mental health products [33].

\section{Set up of quantitative trials with Bosch UK and UCL}

In November 2018, in a qualitative trial with Inmarsat, the British satellite company was set up with their new graduates joining the company. Twenty-two 19-28-year olds were recruited in an email asking for participants for a mental health app well-being study. Over a 5-week course, weekly emails were sent out with qualitative questions as well as a question to prove that the participant had played a level of the course. At trial beginning and end the Adult Resilience Scale (ARS), the Depression test Questionnaire (PHQ 9) and the Generalized Anxiety Disorder 7 (GAD 7) was administered. Fifteen participants remained, leading to a $68 \%$ adherence rate. Though the population was too small to generate statistically valid outcomes, the tendencies cannot be dismissed that showed a decline in depression and anxiety while detecting a rise in resilience. $82 \%$ Of the participants also stated that they used the skills learned in eQuoo within the following week in interpersonal situations, showing that the game has concrete practical applications.

June 24th a 3-arm RCT with over 600 participants at BOSCH UK commenced, sending the Psychological Well-Being (Ryff's Scale) Positive Relationships with others, Resilience (ARS), Personal Growth (PGIS) and a 1-item anxiety likert scale at the beginning, the middle and the end of the 5-week trial.

September a 3-arm RCT will be recruiting amongst 45000 students at UCL London, expecting an average of 4000 participants while administering the Adult Resilience Scale (ARS), the Depression test Questionnaire (PHQ 9) and the Generalized Anxiety Disorder 7 (GAD 7).

\section{Conclusion}

With less than $35 \%$ of the population in developed countries having access to mental health care, it is paramount that we develop easily accessible and affordable tools that help lower the personal and societal burden of mental illness [42]. The 18 28-year-old population that eQuoo is targeting is especially vulnerable and would benefit greatly from a tool they enjoy and is still effective [1]. While thousands of new mobile products target the 3 trillion well-being market, not all of them are safe, effective and compelling. eQuoo may very well be an answer to a tremendous need [43].

\section{References}

1. Donker T, Petrie K, Proudfoot J, Clarke J, Birch MR, et al. (2013) Smartphones for smarter delivery of mental health programs: a systematic review. $J$ Med Internet Res 15: e247. [Crossref]

2. Bakker D, Kazantzis N, Rickwood D, Rickard N (2016) Mental health smartphone apps: review and evidence-based recommendations for future developments. JMIR Ment Health 3: e7. [Crossref]

3. Haugen PT, McCrillis AM, Smid GE, Nijdam MJ (2017). Mental health stigma and barriers to mental health care for first responders: a systematic review and metaanalysis. J Psychiatric Res 94: 218-229. [Crossref]

4. Betterhelp. 2019. Available from: https://www.betterhelp.com/

5. Talkspace. 2019. Available from: https://www.talkspace.com/

6. Crunchbase. 2019. Available from: https://news.crunchbase.com/news/digital-therapystartup-talkspace-raises-50m-in-revolution-led-series-d/

7. Tutty S, Spangler DL, Poppleton LE, Ludman EJ, Simon GE (2010) Evaluating the effectiveness of cognitive-behavioral teletherapy in depressed adults. Behav Ther 41: 229-236. [Crossref]

8. Haas LJ, Benedict JG, Kobos JC (1996) Psychotherapy by telephone: risks and benefits for psychologists and consumers. Prof Psychol Res Pr 27: 154-160.

9. Midkiff DM, Wyatt JW (2008) Ethical issues in the provision of online mental health services (etherapy). J Technology in Human Services, 26: 310-332.

10. Polygon, 2018. Available from: https://www.polygon.com/2018/10/4/17932862/ betterhelp-app-youtube-sponsorship-controversy-explained

11. Calm. 2019. Available from: https://www.calm.com/

12. Headspace. 2019. Available from: https://www.headspace.com/headspace-meditationapp

13. TechCrunch. 2019. Available from: https://techcrunch.com/2019/02/06/meditationapp-calm-hits-unicorn-status-with-fresh-88-million-funding/

14. Headspace. 2019a. Available from: https://www.headspace.com/science

15. Headspace. 2019b. Available from: https://www.headspace.com/science/meditationresearch

16. Perez-De-Albeniz A, Holmes J (2000) Meditation: concepts, effects and uses in therapy. International Journal of Psychotherapy 5: 49-58.

17. Dahiya M (2017) A tool of conversation: Chatbot. International Journal of Computer Sciences and Engineering Open Access 5.

18. Woebot. 2019. Available from: https://woebot.io/

19. Fitzpatrick KK, Darcy A, Vierhile M (2017) Delivering cognitive behaviour therapy to young adults with symptoms of depression and anxiety using a fully automated conversational agent (Woebot): a randomised controlled trial. JMIR Ment Health 4: e19. [Crossref]

20. Tess. 2019. Available from: https://www.x2ai.com/

21. Fulmer R, Joerin A, Gentile B, Lakerink L, Rauws M (2019) Using psychological artificial intelligence (tess) to relieve symptoms of depression and anxiety: a randomized controlled trial. JMIR Ment Health 5: e64. [Crossref] 
22. The Verge. 2016. Available from: https://www.theverge.com/2016/3/24/11297050/ tay-microsoft-chatbot-racist

23. Andersson G, Cuijpers P (2009) Internet-based and other computerized psychological treatments for adult depression: a meta-analysis. Cogn Behav Ther 38: 196-205. [Crossref]

24. Jmango. 2018. Available from: https://jmango360.com/wiki-pages-trends/mobile-appvs-mobile-website-statistics

25. Arnberg FK, Linton SJ, Hultcrantz M, Heintz E, Jonsson U (2014) Internet-delivered psychological treatments for mood and anxiety disorders: a systematic review of their efficacy, safety, and cost-effectiveness. PLoS One 9: e98118. [Crossref]

26. Krupnick JL, Sotsky SM, Simmens S, Moyer J, Elkin I, et al. (1996) The role of the therapeutic alliance in psychotherapy and pharmacotherapy outcome: findings in the national institute of mental health treatment of depression collaborative research program. J Consult Clin Psychol 64: 532-539. [Crossref]

27. Vogel DL, Wade NG, Hackler AH (2007) Perceived public stigma and the willingness to seek counseling: the mediating roles of self-stigma and attitudes toward counseling. Journal of Counseling Psychology 54: 40-50.

28. Huang HY, Bashir M (2017) Users' adoption of mental health apps: examining the impact of information cues. JMIR Mhealth Uhealth 5: $\mathrm{e} 83$.

29. Moodpath. 2019. Available from: https://mymoodpath.com/en/

30. Pacifica. 2019. Available from: https://www.thinkpacifica.com/

31. Pacifica Labs. 2018. Impact of a mobile application (Pacifica) on stress, anxiety, and depression. Available from: https://clinicaltrials.gov/ct2/show/NCT03333707

32. Litvin S, Markus M (2019) How mHealth programmes can treat depression: a randomised controlled trial.
33. Farvolden P, Denisoff E, Selby P, Bagby RM, Rudy L (2005) Usage and longitudinal effectiveness of a web-based self-help cognitive behavioural therapy program for panic disorder. J Med Internet Res 7: e7. [Crossref]

34. Christensen H, Griffiths KM, Korten AE, Brittliffe K, Groves C (2004). A comparison of changes in anxiety and depression symptoms of spontaneous users and trial participants of a cognitive behaviour therapy website. J Med Internet Res 6: e46. [Crossref]

35. Melville KM, Casey LM, Kavanagh DJ (2010) Dropout from internet-based treatment for psychological disorders. Br J Clin Psychol 49: 455-471. [Crossref]

36. Papastergiou M (2009) Exploring the potential of computer and video games for health and physical education: A literature review. Computers and Education 53: 603-622.

37. Roepke AM, Jaffee SR, Riffle OM, McGonigal J, Broome R, et al. (2015) Randomized controlled trial of SuperBetter, a smartphone-based/internet-based self-help tool to reduce depressive symptoms. Games Health J 4: 235-246. [Crossref]

38. Zimmerman M, Balling C, Chelminski I, Dalrymple K (2018) Understanding the severity of depression: which symptoms of depression are the best indicators of depression severity? Compr Psychiatry 87: 84-88. [Crossref]

39. Svenson S (2004) Carrier-based drug delivery. ACS Symposium Series, 879, Chapter 1: 2-23. American Chemical Society.

40. Fleming TM, Bavin L, Stasiak K, Hermansson-Webb E, Merry SN, et al. (2017) Serious games and gamification for mental health: Current status and promising directions. Front Psychiatry 7: 215. [Crossref]

41. eQuoo. 2019. Available from: https://www.equoogame.com

42. Community Mental Health Survey. 2017. Available from: https://www.cqc.org.uk/ sites/default/files/20180515_cmh17_statisticalrelease.pdf

43. Global Wellness Institute. 2018. Available from: https://globalwellnessinstitute.org/ press-room/statistics-and-facts/

Copyright: (C2019 Litvin S. This is an open-access article distributed under the terms of the Creative Commons Attribution License, which permits unrestricted use, distribution, and reproduction in any medium, provided the original author and source are credited. 